\title{
Tissue-specific expression and regulation of sexually dimorphic genes in mice
}

\author{
Xia Yang, ${ }^{1}$ Eric E. Schadt, ${ }^{2}$ Susanna Wang, ${ }^{3}$ Hui Wang,${ }^{4}$ Arthur P. Arnold, ${ }^{5}$ \\ Leslie Ingram-Drake, ${ }^{3}$ Thomas A. Drake, ${ }^{6}$ and Aldons J. Lusis ${ }^{1,3,7}$ \\ ${ }^{1}$ Department of Medicine, David Geffen School of Medicine, University of California, Los Angeles, California 90095, USA; ${ }^{2}$ Rosetta \\ Inpharmatics, LLC, a Wholly Owned Subsidiary of Merck \& Co. Inc., Seattle, Washington 98109, USA; ${ }^{3}$ Department of Human \\ Genetics, University of California, Los Angeles, California 90095, USA; ${ }^{4}$ Department of Statistics, College of Letters and Science, \\ University of California, Los Angeles, California 90095, USA; ${ }^{5}$ Department of Physiological Science, and Laboratory of \\ Neuroendocrinology of the Brain Research Institute, University of California, Los Angeles, California 90095, USA; ${ }^{6}$ Department of \\ Pathology and Laboratory Medicine, University of California, Los Angeles, California 90095, USA
}

\begin{abstract}
We report a comprehensive analysis of gene expression differences between sexes in multiple somatic tissues of 334 mice derived from an intercross between inbred mouse strains $\mathrm{C} 57 \mathrm{BL} / 6$ ] and $\mathrm{C} 3 \mathrm{H} / \mathrm{He}$ ). The analysis of a large number of individuals provided the power to detect relatively small differences in expression between sexes, and the use of an intercross allowed analysis of the genetic control of sexually dimorphic gene expression. Microarray analysis of 23,574 transcripts revealed that the extent of sexual dimorphism in gene expression was much greater than previously recognized. Thus, thousands of genes showed sexual dimorphism in liver, adipose, and muscle, and hundreds of genes were sexually dimorphic in brain. These genes exhibited highly tissue-specific patterns of expression and were enriched for distinct pathways represented in the Gene Ontology database. They also showed evidence of chromosomal enrichment, not only on the sex chromosomes, but also on several autosomes. Genetic analyses provided evidence of the global regulation of subsets of the sexually dimorphic genes, as the transcript levels of a large number of these genes were controlled by several expression quantitative trait loci (eQTL) hotspots that exhibited tissue-specific control. Moreover, many tissue-specific transcription factor binding sites were found to be enriched in the sexually dimorphic genes.
\end{abstract}

[Supplemental material is available online at www.genome.org. The microarray data from this study have been deposited to GEO under accession nos. GSE2814, GSE3086, GSE3087, and GSE3088.]

Mammalian sexual differentiation, initiated by the presence or absence of the testis-determining factor encoded on the Y chromosome (SRY in humans and Sry in mice) and mediated by the effects of sex hormones, has been studied in great detail (Smith 1994; McElreavey et al. 1995; Vilain and McCabe 1998; Hughes 2001; Arnold 2004). It is clear that the regulatory pathways underlying sexual differentiation result in extensive differences in gene expression in adults. Briefly, tens to thousands of genes were shown to be sexually dimorphic in mouse liver, kidney, blastocysts, lacrimal gland, prenatal brain, and adult brain substructures (Lauber et al. 1991; Anderson 2002; Xu et al. 2005; Dewing et al. 2003; Rinn et al. 2004; Vawter et al. 2004; AmadorNoguez et al. 2005; Clodfelter et al. 2006; Kobayashi et al. 2006; Richards et al. 2006). However, because of the limited power resulting from relatively small sample sizes in most studies, the extent and tissue specificity of sexual dimorphism in gene expression have not been fully explored. In addition, the genetic and transcriptional regulatory mechanisms underlying the sex differences in gene expression have been investigated primarily in liver (Tullis et al. 2003; Waxman and Celenza 2003; Wiwi and Waxman 2004; Clodfelter et al. 2006), and, thus, the regulatory mechanisms involved in other tissues remain poorly understood. Since nearly all common diseases exhibit some degree of sex bias,

${ }^{7}$ Corresponding author.

E-mail jlusis@mednet.ucla.edu; fax (310) 794-7345.

Article published online before print. Article and publication date are at http:// www.genome.org/cgi/doi/10.1101/gr.5217506. Freely available online through the Genome Research Open Access option. often very dramatic (Spitzer 1999; Wizemann and Pardue 2001; Depner 2003), a systematic understanding of the mechanisms underlying physiological differences between sexes is of great importance. Such an understanding could also be useful in explaining sex differences in drug metabolism.

Here we present a general survey of sexually dimorphic genes in mouse liver, adipose, whole brain, and muscle and provide evidence for tissue-specific expression and regulation of these genes. Our results revealed a surprisingly large number of significant differences in gene expression between the sexes, ranging from a few hundred to more than ten thousand in the different tissues examined. The overlap in the sexually dimorphic genes in the four tissues examined was minimal, and there were striking differences in the identities and functional categories of the genes between tissues. Many of the genes that we identified have been implicated in various common diseases in which disease susceptibility is sex-biased. Our results also revealed chromosomal enrichment as well as extensive tissuespecific regulation of sexually dimorphic genes. The experimental design of our study, involving a genetic intercross, allowed us to map expression quantitative trait loci (eQTL) hotspots controlling large numbers of sexually dimorphic genes.

\section{Results}

Experimental design

Previous studies of sexual dimorphism in mice have primarily utilized inbred strains. Although such a design is more straight- 
forward, it does not provide insights into genetic regulation of the sexually dimorphic gene expression. To overcome this limitation and introduce genetic perturbations necessary for the identification of genetic regulation, we chose to examine mice derived from an $F_{2}$ intercross between two common but not closely related laboratory inbred strains, $\mathrm{C} 3 \mathrm{H} / \mathrm{HeJ}(\mathrm{C} 3 \mathrm{H})$ and $\mathrm{C} 57 \mathrm{BL} / 6 \mathrm{~J}$ (B6). $\mathrm{C} 3 \mathrm{H}$ and $\mathrm{B} 6$ have been previously shown to exhibit differences in numerous traits influenced by sex, including adiposity, plasma lipid metabolism, and susceptibility to atherosclerosis (Lusis et al. 1983; Paigen et al. 1987; Machleder et al. 1997; Taylor et al. 1999). Therefore, our study also had the potential to explore the influence of sex on genetic regulation of these clinical and physiological phenotypes. For example, we have recently identified multiple quantitative trait loci (QTL) with sex interactions for a fat mass trait using this cross (Wang et al. 2006). Our ultimate goal is to link specific sexually dimorphic genes or pathways with specific traits influenced by sex. Because of their potential relevance to the common diseases and physiological processes such as atherosclerosis, adiposity, lipid metabolism, drug response, and neurological and psychiatric diseases, we chose to examine four somatic tissues-liver, adipose, whole brain, and muscle-using global microarray analysis.

The ability to accurately identify differences in gene expression depends on the number of independent samples examined and on the extent of genetic and environmental variation. In order to have the statistical power to identify subtle differences in gene expression between male and female mice, we examined a very large set of animals, 169 females and 165 males. Although the genetic variation in an $F_{2}$ intercross is larger than that in inbred mice, the substantially increased sample size used in our study overcomes the negative effect of the increased variation on the power to detect differences between sexes, as demonstrated by the power curves (Supplemental Fig. 1; Supplemental Methods). For example, at the $P<0.01$ significance level, a sample size of $n=167$ has $>90 \%$ power to detect a $5 \%$ mean difference between males and females in an $F_{2}$ population with a withingroup variance of 0.4 ; whereas when using a smaller sample size of $n=10$, the power would be $<10 \%$ in inbred mice with a much smaller within-in group variance of 0.2 .

\section{Degree of sexually dimorphic gene expression in four somatic} tissues

An unsupervised, two-dimensional hierarchical clustering analysis of the microarray data of the $\mathrm{B} 6 \times \mathrm{C} 3 \mathrm{H} F_{2}$ progeny revealed striking gender effects on gene expression (Supplemental Methods). As exemplified in Figure 1 , the mean $\log _{10}$ (expression ratio of sample vs. pool) values of the 1332 transcripts identified as being most transcriptionally active (see definition in Methods) in liver were clustered in all the mice studied. The visually distinct expression patterns from several gene clusters clearly separate the mice into two major groups (females and males) and an outlier group containing a few mice of both genders. The outlier group was separated mainly based on the patterns of a few small gene clusters with strong signals that showed similar expression in the two major mouse groups, possibly owing to tissue contamination or microarray artifacts. Nevertheless, even in this outlier group, the mice were separated into females and males without any overlap based on the expression pattern of the remaining genes. Similar distinct sexually dimorphic gene clustering was also identified in adipose and muscle, but to a much lesser extent in brain (Supplemental Fig. 2).

Using the nonparametric Wilcoxon test and the parametric Student's $t$-test, we analyzed selected "active genes" that represent expressed genes in individual tissues for differential gene expression between male and female mice (see Methods for rationale and procedure for active gene selection). Here we define the differentially expressed genes between sexes as "sexually dimorphic" genes. The genes that are expressed more highly in females are termed "female-biased" genes, and those expressed more highly in males are called "male-biased" genes. Both the $t$-test and the Wilcoxon test identified similar sets of sexually dimorphic genes. In this manuscript, we report the genes identified using the nonparametric Wilcoxon test to avoid issues related to the normality assumptions underlying the $t$-test. Table 1 summarizes the distribution of genes that were significantly differentially expressed between sexes at various stringency levels (fold change $>1,>1.2,>2$, or $>3 ; P<0.01$ ). As discussed below, sexually dimorphic genes varying at different fold-change thresholds demonstrated distinct biological properties, thus supporting the necessity for such multilevel analyses. The complete lists of all sexually dimorphic genes in individual tissues are available in Supplemental Tables 1-4.

\section{Genome Research}

www.genome.org 
Table 1. Distribution of genes that are differentially expressed between males and females in liver, adipose, muscle, and brain $(P<0.01)$

\begin{tabular}{|c|c|c|c|c|c|}
\hline Tissue & $\begin{array}{l}\text { Active } \\
\text { genes }\end{array}$ & $\begin{array}{c}\text { Fold } \\
\text { change }\end{array}$ & Dimorphic genes & Female-biased & Male-biased \\
\hline \multirow[t]{4}{*}{ Liver } & \multirow[t]{4}{*}{12,845} & $>1$ & 9250 (72.0\%) & 4809 (37.4\%) & 4441 (34.6\%) \\
\hline & & $>1.2$ & $1731(13.5 \%)$ & $737(5.7 \%)$ & $994(7.7 \%)$ \\
\hline & & $>2$ & $142(1.1 \%)$ & $61(0.5 \%)$ & $81(0.6 \%)$ \\
\hline & & $>3$ & $65(0.5 \%)$ & $30(0.2 \%)$ & $35(0.3 \%)$ \\
\hline \multirow[t]{4}{*}{ Adipose } & \multirow[t]{4}{*}{16,664} & $>1$ & $11,336(68.0 \%)$ & $6358(38.2 \%)$ & 4978 (29.9\%) \\
\hline & & $>1.2$ & $3240(19.4 \%)$ & $1521(9.1 \%)$ & $1719(10.3 \%)$ \\
\hline & & $>2$ & $215(0.8 \%)$ & $120(0.7 \%)$ & $95(0.6 \%)$ \\
\hline & & $>3$ & $47(0.3 \%)$ & $31(0.2 \%)$ & $16(0.1 \%)$ \\
\hline \multirow[t]{4}{*}{ Muscle } & \multirow[t]{4}{*}{7367} & $>1$ & $4083(55.4 \%)$ & 2275 (30.9\%) & $1808(24.5 \%)$ \\
\hline & & $>1.2$ & 716 (9.7\%) & $382(5.2 \%)$ & 334 (4.5\%) \\
\hline & & $>2$ & $45(0.6 \%)$ & $13(0.2 \%)$ & $32(0.4 \%)$ \\
\hline & & $>3$ & $9(0.1 \%)$ & $2(0.0 \%)$ & $7(0.1 \%)$ \\
\hline \multirow[t]{4}{*}{ Brain } & \multirow[t]{4}{*}{4508} & $>1$ & $612(13.6 \%)$ & $355(7.9 \%)$ & $257(5.7 \%)$ \\
\hline & & $>1.2$ & $34(0.8 \%)$ & $18(0.4 \%)$ & $16(0.4 \%)$ \\
\hline & & $>2$ & $6(0.1 \%)$ & $1(0.0 \%)$ & $5(0.1 \%)$ \\
\hline & & $>3$ & $3(0.1 \%)$ & $0(0.0 \%)$ & $3(0.1 \%)$ \\
\hline \multirow[t]{4}{*}{ All four tissues } & \multirow[t]{4}{*}{1768} & $>1$ & $27^{\mathrm{a}}(1.5 \%)$ & $14(0.8 \%)$ & $13(0.7 \%)$ \\
\hline & & $>1.2$ & $8^{\mathrm{a}}(0.5 \%)$ & $1(0.1 \%)$ & $7(0.4 \%)$ \\
\hline & & $>2$ & $3^{\mathrm{a}}(0.2 \%)$ & $0(0.0 \%)$ & $3(0.2 \%)$ \\
\hline & & $>3$ & $3^{\mathrm{a}}(0.2 \%)$ & $0(0.0 \%)$ & $3(0.2 \%)$ \\
\hline
\end{tabular}

The percentage of sexually dimorphic genes in total active genes for the corresponding tissues is in parenthesis.

${ }^{\mathrm{a} C}$ Consistent direction in all four tissues.

At the $P<0.01$ significance level, 9250, 11,336, 4083, and 612 genes demonstrated sexually dimorphic expression in liver, adipose, muscle, and brain, respectively. Based on our algorithm for the identification of genes with significant expression signals, these numbers represented $72.0 \%$ (liver), $68.0 \%$ (adipose), 55.4\% (muscle), and $13.6 \%$ (brain) of the active genes. Using Storey's Q-value approach (Storey and Tibshirani 2003), the false discovery rates (FDR) of the sexually dimorphic genes in liver, adipose, muscle, and brain were estimated to be $0.2 \%, 0.2 \%, 0.5 \%$, and $5 \%$, respectively, supporting the accuracy of our estimates of the numbers of genes that are sexually dimorphic. The much lower level of apparent sexual dimorphism in brain as compared to other tissues was also observed when different fold-change levels were taken into account.

Many of the sexually dimorphic genes $(81.3 \%$ for liver, $71.4 \%$ for adipose tissue, $82.5 \%$ for muscle, and $94.4 \%$ for brain) displayed $<1.2$-fold difference in expression between males and females. The range, mean, and median of the fold change for the sexually dimorphic, female-biased, and male-biased genes are summarized in Supplemental Table 5. Both the fold-change mean and median also indicated that although many genes are sexually dimorphic, most sex differences in gene expression are modest in size $(<1.2$-fold $)$.

Tissue specificity of sexually dimorphic genes and enriched functional categories

We compared both the active gene sets and the sexually dimorphic genes across tissues in order to assess tissue specificity. Figure $2 \mathrm{~A}$ shows that there exists a considerable overlap in the active genes between any two tissues. However, there was a significantly smaller overlap in the sexually dimorphic genes as compared to the active genes across tissues $(P<2 \mathrm{e}-16$ as determined by a test for equality of proportions for each overlapping region) (Fig. 2B cf. 2A). Thus, clearly, sexually dimorphic genes are highly tissue-specific.
As shown in Table 2 (see Supplemental Table 6 for more details), there were striking differences in the overrepresented functional categories for the sexually dimorphic genes in individual tissues. The only common functionalities were steroid and lipid metabolism, oxidoreductase activity, and defense response between liver and adipose tissues. Another observation is that there were very few overlaps in the enriched pathways between genes varied at different fold-change levels for the same tissue, highlighting the importance of analyzing genes by fold-change thresholds.

The sexually dimorphic genes in liver were enriched for protease inhibitor activity, immune/defense response, carboxylic acid, fatty acid, steroid and lipid metabolic pathways, electron transport, monooxygenase activity, and oxidoreductase activity. These genes included cytochrome P450s (Cyp450), flavin-containing monooxygenases, sulfotransferases, hydroxysteroid dehydrogenases, CD antigens, chemokine ligands, complement components, serum amyloids, interleukins, and various others. Most liver sexually dimorphic genes and pathways identified in previous studies were confirmed here, and 7000 genes represented novel findings (Rinn et al. 2004; Amador-Noguez et al. 2005; Clodfelter et al. 2006).

The sexually dimorphic genes identified in adipose tissue were enriched for the functions of ion transport, immune response, oxidoreductase activity, cell motility, morphogenesis/ organogenesis, muscle contraction/development, and lipid and steroid hormone metabolism. These genes encode annexins, actinins, cadherins, calcium channel subunits, solute carriers, procollagens, Cyp450s, hydroxysteroid dehydrogenases, cytochrome $c$ oxidase subunits, chemokine receptors and ligands, complement components, FC receptors, histocompatibility proteins, myosin chains, and troponins. The finding that genes involved in muscle contraction/development were also overrepresented may have resulted from contamination introduced during tissue collection and anatomical differences in the gonadal fat pads between sexes. Another intriguing possibility is that a dual function may exist for these muscle-related genes; for example, they could conceivably function in energy expenditure in adipose tissue.

For the sexually dimorphic genes in muscle, functional categories such as ribosome biosynthesis/assembly and translation (represented by a large number of female-biased genes encoding ribosomal proteins) and polyamine metabolism (represented by male-biased genes encoding spermine oxidase, ornithine decarboxylase, $s$-adenosylmethione decarboxylase, and others) were found to be overrepresented. Genes involved in muscle contraction and development, such as myosin chains, troponins, actin, and dystrophin, were also found to be enriched in the femalebiased genes. These findings are somewhat intriguing since there is no obvious reason for enhanced protein synthesis and muscle development processes in females. Studies on the effects of estrogens on muscle development have been somewhat contradictory (Kahlert et al. 1997; McCormick et al. 2004), but there is 
A

\section{Overlap of active genes}

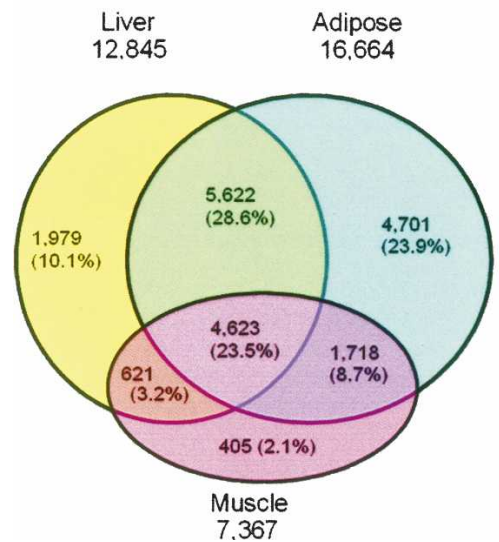

\section{B}

Overlap of sexually dimorphic genes

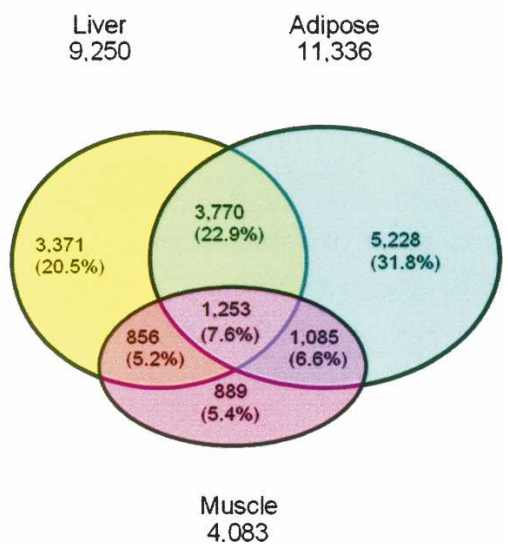

Figure 2. Venn diagram showing tissue specificity of the active genes and the sexually dimorphic genes. (A) Overlap of active genes among liver, adipose, and muscle. (B) Overlap of sexually dimorphic genes among liver, adipose, and muscle. The gene number in each distinct area and its percentage over the combined total gene number $(19,669$ for $A$ and 16,452 for $B)$ are shown.

in vivo radioimmunoassays (DeVito 1988) and in cultures of embryonic hypothalamic cells (Beyer et al. 1992). It encodes a hormone expressed in the pituitary gland and hypothalamus, and has been suggested to be a neuropeptide that modulates reproductive behavior such as the onset of maternal behavior (Mann and Bridges 2001). In addition to several genes previously identified in brain substructures, we observed $\sim 600$ novel sexually dimorphic genes in brain. These sex differences could help explain sex differences in the incidence or progression of neurological and psychiatric diseases.

Across all four tissues profiled, a total of 71 genes displayed differential expression between sexes, but only 27 showed consistent direction, either female-biased in all tissues or male-biased in all tissues (Table 1; Supplemental Table 7). Although we failed to identify any overrepresented functional category evidence indicating that estradiol-17 $\beta$ increases skeletal muscle protein degradation (Hayden et al. 1992). Thus, the observed female-biased expression of genes involved in protein translation may be a compensation for such an enhanced degradation process.

In the brain, the only functional category enriched for the entire set of sexually dimorphic genes was RNA helicase activity, such as $D d x 3 x / D b x$ (female-biased) and $D d x 3 y / D b y$ (male-biased). Defense response genes encoding chemokine ligands, heat shock proteins, and histocompatibility proteins were overrepresented in the male-biased genes. For female-biased genes, JAK-STAT cascade and lactation pathways were enriched owing to the presence of a single gene, prolactin ( $P r l)$. Prl has been previously shown to be expressed more highly in females in rat brain using in these transcripts, we noticed that among the 27 genes, four were located on the $\mathrm{Y}$ chromosome and three were on the $\mathrm{X}$ chromosome.

Tissue-independent sex chromosome enrichment and tissue-specific autosome enrichment

Sexually dimorphic genes such as those involved in sex development and differentiation as well as those preferentially expressed in reproductive tissues have been found to be concentrated on sex chromosomes, particularly the $\mathrm{X}$ chromosome (Saifi and Chandra 1999; Wang et al. 2001; Arnold 2004; Vawter et al. 2004). For the four somatic tissues studied here, Table 3 summarizes the chromosomes on which all sexually dimorphic genes,

Table 2. Comparison of the enriched functional categories in the sexually dimorphic genes in liver, adipose, muscle, and brain

\begin{tabular}{|c|c|c|c|c|}
\hline $\begin{array}{l}\text { Fold } \\
\text { change }\end{array}$ & Liver & Adipose & Muscle & Brain \\
\hline$>1$ & & & $\begin{array}{l}\text { Ribosome biosynthesis/assembly } \\
\text { RNA binding } \\
\text { Translation }\end{array}$ & \\
\hline$>1.2$ & $\begin{array}{l}\text { Protease inhibitor } \\
\text { Steroid hydroxylase } \\
\text { Defense response } \\
\text { Immune response } \\
\text { Carboxylic acid metabolism } \\
\text { Fatty acid metabolism }\end{array}$ & $\begin{array}{l}\text { Calcium/metal ion binding } \\
\text { lon cation transporter } \\
\text { Oxidoreductase activity } \\
\text { Immune response } \\
\text { Cell motility/adhesion }\end{array}$ & & RNA helicase activity \\
\hline $\begin{array}{l}>2 \\
>3\end{array}$ & $\begin{array}{l}\text { Electron transport } \\
\text { Monoxygenase activity } \\
\text { Oxidoreductase activity } \\
\text { Lipid metabolism } \\
\text { Steroid biosynthesis } \\
\text { Steroid metabolism } \\
\text { Serine-type endopeptidase } \\
\text { inhibitor activity }\end{array}$ & $\begin{array}{l}\text { Morphogenesis } \\
\text { Organogenesis } \\
\text { Muscle contraction } \\
\text { Muscle development } \\
\text { Oxidoreductase activity } \\
\text { Lipid metabolism } \\
\text { C21 steroid hormone biosynthesis } \\
\text { C21 steroid hormone metabolism } \\
\text { Hormone biosynthesis/ metabolism } \\
\text { Androgen and estrogen metabolism }\end{array}$ & $\begin{array}{l}\text { Polyamine metabolism } \\
\text { Biogenic amine metabolism } \\
\text { Spermine metabolism }\end{array}$ & $\begin{array}{l}\text { ATP-dependent RNA helicase } \\
\text { activity } \\
\text { RNA dependent ATPase activity }\end{array}$ \\
\hline
\end{tabular}

Functional categories with $P<0.05$ (Fisher's exact test with Bonferroni correction) at various fold-change thresholds are summarized and shown. A detailed listing of all enriched pathways and associated $P$-values are available in Supplemental Table 6 . The gene categories in common between tissues are highlighted in bold.

\section{Genome Research}

www.genome.org 
Tissue specificity of sexual dimorphism

Table 3. Overrepresented chromosomes for the sexually dimorphic genes (All), female-biased genes (F), and male-biased genes (M) compared to the active genes in liver, adipose, muscle, and brain

\begin{tabular}{|c|c|c|c|c|c|c|c|c|c|c|c|c|}
\hline \multirow[b]{2}{*}{ Fold change } & \multicolumn{3}{|c|}{ Liver } & \multicolumn{3}{|c|}{ Adipose } & \multicolumn{3}{|c|}{ Muscle } & \multicolumn{3}{|c|}{ Brain } \\
\hline & All & $F$ & M & All & $F$ & M & All & $F$ & M & All & $F$ & M \\
\hline$>1$ & $x$ & $10, x, 17$ & $\mathrm{Y}$ & 7,15 & 7,15 & 3,10 & $x$ & $\mathbf{x}$ & $\mathrm{Y}, 10$ & $X, Y, 14,5$ & $\mathbf{x}, 14$ & $\mathbf{Y}, \mathrm{X}, 5,17$ \\
\hline$>1.2$ & 3,19 & 19 & $Y, 3,18$ & $3, X, Y, 10$ & & $Y, 3$ & $\mathrm{Y}, 14$ & $\mathbf{x}, 2$ & $\mathbf{Y}, 1$ & $\mathbf{X}, \mathbf{Y}$ & $\mathbf{x}, 13$ & $\mathbf{Y}, \mathbf{X}$ \\
\hline$>2$ & $19, Y, 12$ & 19,5 & $\mathbf{Y}, 18$ & $\mathbf{Y}, 3$ & 3,15 & $\mathbf{Y}, 1,10$ & $\mathbf{Y}$ & & $\mathbf{Y}$ & $\mathbf{Y}$ & 13 & $\mathbf{Y}$ \\
\hline$>3$ & $\mathbf{Y}, 19$ & 19 & $\mathbf{Y}, 18$ & $\mathbf{Y}, 13$ & 13 & $\mathbf{Y}$ & $\mathbf{Y}, 2$ & 2 & $\mathbf{Y}$ & $\mathbf{Y}$ & & $\mathbf{Y}$ \\
\hline
\end{tabular}

The overrepresented chromosomes with $P<0.05$ determined by Fisher's exact test are listed, and those with $P<0.05$ after Bonferroni correction for multiple chromosome comparisons are highlighted in bold.

female-biased as well as male-biased genes, are enriched $(P<0.05$, Fisher's exact test; a detailed listing of the $P$-values can be found in Supplemental Table 8). When only considering the highly overrepresented chromosomes at any fold-change level $(P<0.05$ after Bonferroni correction for multiple chromosome comparisons), the sexually dimorphic genes in brain were significantly enriched on the $\mathrm{X}$ chromosome, and the dimorphic genes for all four tissues were highly enriched for Y chromosome genes. Additional overrepresented loci included chromosome 19 for liver and chromosome 2 for muscle. An analysis of the femalebiased genes revealed enrichment on chromosome 19 for liver, chromosome 7 for adipose, the $\mathrm{X}$ chromosome and chromosome 2 for muscle, and the X chromosome and chromosome 13 for brain. The male-biased genes were enriched on the $\mathrm{X}$ chromosome for brain and the Y chromosome for all four tissues. Thus, we not only confirmed a concentration of sexually dimorphic transcripts on sex chromosomes in various somatic tissues, but observed tissue-specific autosomal localization.

We hypothesized that the observed tissue-specific enrichment of sexually dimorphic genes on autosomes might reflect the enrichment of sexually dimorphic genes with similar functionalities. An analysis of liver dimorphic genes on the overrepresented chromosome 19 revealed an enrichment for oxidoreductase activity and monooxygenase activity $(P=4.8 \mathrm{e}-6$ and 7.7e-5, respectively, by Fisher's exact test; both with FDR $<5 \%$ ). Many of these chromosome 19 genes were Cyp450s, a wellknown group of sexually dimorphic genes in liver that are important for drug and lipid metabolism (Rinn et al. 2004; Wiwi and Waxman 2004; Amador-Noguez et al. 2005). The femalebiased adipose genes on chromosome 7 were enriched for ion channels and muscle contraction activity $(P=1.4 \mathrm{e}-4$ and $P=9.2 \mathrm{e}-4$, Fisher's exact test, respectively; both with FDR $<5 \%$ ). In both cases, a close relationship between autosomal enrichment and functional enrichment was established. Such relationship was weaker for other overrepresented autosomes.

Tissue-specific enrichment of transcription factor binding sites (TFBS)

As an approach to understanding the regulation of sexually dimorphic gene expression, we searched for known TFBS in the conserved sequences within 5000-bp upstream and downstream regions of the sexually dimorphic genes. Using the oPPOSUM program, which contains $\sim 100$ verified TFBS in its database, the enrichment for TFBS was analyzed, and a tissue-specific pattern in TFBS enrichment was observed. When all sexually dimorphic genes in individual tissues were considered, only brain genes showed significant enrichment of TFBS for four transcription factors, namely, MEF2, Pax6, NF-Y, and SAP-1 $(P<0.05$, Fisher's exact test). Among them, NF-Y has been associated with estrogen receptors (Wang et al. 1999; Farsetti et al. 2001). At a fold-change level $>1.2$, several overrepresented TFBS were identified in adipose, liver, and muscle (Supplemental Tables 13-15). Among these, only TFBS for five transcription factors (SRY, PBF, MNB1A, Dof2, and Nkx) were shared in these three tissues. It is interesting to note that the binding sites for SRY and Nkx were among these five TFBS. Nkx factors are known to be regulated by androgens and are responsible for hypothalamic morphogenesis and the neuroendocrine process of sexual development (Bieberich et al. 1996; Lee et al. 2001). There was no overlap in the enriched TFBS between brain and any of the other tissues tested. It is important to note that only the analyses for adipose and muscle TFBS demonstrated FDR $<5 \%$ at the $P<0.05$ level; therefore, the findings and interpretation on liver and brain TFBS have to be taken with caution.

Tissue-specific genetic regulation of sexually dimorphic genes: Analysis of expression quantitative trait loci (eQTL)

To determine if the sexually dimorphic genes were under specific localized genetic control, we performed linkage analysis to identify significant and suggestive eQTL (loci controlling transcript levels; detailed definition in Methods) and searched for hotspots that were enriched for eQTL. As shown in Table 4, a total of 11 loci were identified for liver, and six were found for adipose. The genomewise eQTL distribution and the localization of the hotspots are depicted in Figure 3 (for significant eQTL) and Supplemental Figure S3 (for suggestive eQTL). There was no overlap in the loci between the two tissues. We did not find clustering of either significant or suggestive eQTL for the sexually dimorphic genes in muscle and brain. These results indicate that genetic regulation of the tissue-specific sexual dimorphism is also tissue-specific.

Interestingly, we found that in several cases, sexually dimorphic genes with similar functions were regulated by specific genetic loci (Table 4). For example, the sexually dimorphic genes in adipose with significant eQTL at the chromosome 1 marker rs3694065 were highly enriched for defense response and intracellular signaling cascade pathways ( $P=4.81 \mathrm{e}-3$ and $2.77 \mathrm{e}-2$, respectively; Fisher's exact test after Bonferroni correction for multiple pathways). Sexually dimorphic genes in liver with significant eQTL at marker rs3699728 on chromosome 5, on the other 
Yang et al.

Table 4. eQTL hotspots with significantly enriched significant and/or suggestive eQTL in adipose and liver

\begin{tabular}{|c|c|c|c|c|}
\hline Tissue & $\begin{array}{l}\text { SNP } \\
\text { name }\end{array}$ & Chromosome & $\begin{array}{c}\mathrm{cM} \\
\text { position }\end{array}$ & Enriched functional categories/chromosomes \\
\hline Adipose & rs $3694065^{a}$ & 1 & 50.4 & Defense response; intracellular signaling cascade \\
\hline Adipose & rs3699539a & 12 & 38.2 & Lysosome; hydrogen ion transporter activity \\
\hline Adipose & rs3701757 & 13 & 0.7 & Steroid synthesis/metabolism \\
\hline Adipose & rs $3707560^{\mathrm{a}}$ & 14 & 27.1 & $\begin{array}{l}\text { Chemokine activity; G-protein-coupled receptor binding; defense response; immune response; } \\
\text { inflammatory response }\end{array}$ \\
\hline Adipose & rs3708614 & 15 & 30.7 & chromosome 15; striated muscle thin filament \\
\hline Adipose & rs3708392 & 19 & 46.7 & \\
\hline Liver & rs3691092 & 1 & 8.0 & \\
\hline Liver & rs3695988 & 1 & 11.1 & Ion transporter activity; oxidative phosphorylation; mitochondrion \\
\hline Liver & rs3709457 & 1 & 13.3 & Transferase activity/transfer sulfur-containing groups \\
\hline Liver & rs3660030 & 3 & 35.3 & chromosome 3; defense response; immune response \\
\hline Liver & rs3704040 & 4 & 43.9 & chromosome 4 \\
\hline Liver & rs3714117 & 4 & 65.5 & chromosome 4 \\
\hline Liver & rs3699728 a & 5 & 48.5 & Cell cycle; cell proliferation; DNA replication and chromosome cycle \\
\hline Liver & rs4138743 & 5 & 35.3 & chromosome 5 \\
\hline Liver & rs3713031 & 7 & 25.8 & chromosome 7 \\
\hline Liver & rs3686437 & 12 & 189.6 & chromosome 12 \\
\hline Liver & rs3681284 & 13 & 30.4 & chromosome 13 \\
\hline
\end{tabular}

Significantly enriched functional categories or chromosomes for genes with eQTL at the marker locations are reported. The significance level is $P<0.05$, Fisher's exact test with Bonferroni correction.

aFor both significant and suggestive eQTL. Other unlabeled markers are for suggestive eQTL only.

hand, were enriched for cell cycle, cell proliferation, DNA replication, and chromosome cycle pathways (Bonferroni-corrected $P=5.14 \mathrm{e}-4,1.44 \mathrm{e}-2,1.91 \mathrm{e}-2$, respectively). These results suggest that sexually dimorphic genes are regulated partially as independent functional subsets specific to a given tissue.

About half of the eQTL hotspots controlled the expression of multiple sexually dimorphic genes in a local manner; that is, the subset of genes regulated by the eQTL mostly mapped on the same chromosome as the hotspot itself. Whether this regulation occurs in cis, as in the $\beta$-globin locus control region, or in trans, requires further investigation.

Among the 17 loci, four (one for liver and three for adipose) were identified when either significant or suggestive eQTL were considered and 13 (10 for liver and three for adipose) were found only when suggestive eQTL were used. We focused on the four loci enriched for both significant and suggestive eQTL for the sexually dimorphic genes, namely, rs3707560 (chromosome 14, $27.1 \mathrm{cM}$ ), rs3699539 (chromosome 12, $38.2 \mathrm{cM}$ ) and rs3694065 (chromosome 1, $50.4 \mathrm{cM}$ ) for adipose, and rs3699728 (chromosome $5,48.5 \mathrm{cM}$ ) for liver. Genes with eQTL at these loci are listed in Supplemental Tables 12-15. We searched $10 \mathrm{Mb}$ upstream and downstream of these loci in order to identify candidate regulatory genes. Each locus contained hundreds of annotated genes. We reason that the candidate genes themselves should show differential expression between sexes in order to regulate other sexually dimorphic genes. This narrowed the list down to 99,62 , 38, and 55 genes for adipose loci rs3707560, rs3699539, rs3694065, and liver hotspot rs3699728, respectively (Supplemental Tables 16-19). In addition, a total of 10 genes exhibited significant or suggestive cis-eQTL (that is, the genes whose eQTL map to the gene encoding the transcript) at these loci (Supplemental Tables 16-19). These genes represented attractive candidates.

\section{Discussion}

Differential gene expression between sexes has been observed for a significant portion of the genes in the transcriptomes of various organisms, despite nearly identical genome sequences between males and females. Here, we report surprisingly wide-spread sexually dimorphic gene expression in mice, as manifested by the identification of thousands of differentially expressed genes between males and females in three of the four somatic tissues analyzed, brain being the exception. The degree of sexual dimorphism ranged from $\sim 14 \%$ (brain) to $\sim 70 \%$ (liver) of actively expressed genes in various tissues. Since brain is a highly heterogeneous tissue and striking physiological differences occur between sexes in specific brain regions (Arnold 2004), sex differences in gene expression within individual regions of the brain may well be masked when whole brain is studied. Because of this, and the limited sensitivity of microarrays to detect low levels of gene expression, it is likely that we are underestimating the number of sexually dimorphic genes in the brain.

It is important to note that $70 \%$ 95\% of the sexually dimorphic genes demonstrated fold changes of $<1.2$ in individual tissues. Most molecular studies of sexual dimorphism have focused on genes exhibiting large differences in expression between sexes. While these are likely to be the genes important for sexspecific physiologic functions, a large number of genes with small differences between sexes could well contribute to sexbiased susceptibility to common diseases such as atherosclerosis, diabetes, autoimmune disease, and many others.

The sexually dimorphic genes were found to be highly tissue-specific, as indicated by the minimal overlap in gene identities and the striking differences in most enriched pathways in different tissues. Most ontological categories identified for the sexually dimorphic genes are highly relevant to the distinct functions of the individual tissues as well as the diseases that affect the specific tissues.

Previous studies on sex and reproductive tissues suggested that sexually dimorphic genes are concentrated on sex chromosomes, particularly the X chromosome (Saifi and Chandra 1999; Wang et al. 2001; Arnold 2004; Vawter et al. 2004). Here, we confirmed such a sex-chromosome enrichment in various somatic tissues. This phenomenon could be explained by the unusual sexually dimorphic selection pressures experienced by

\section{Genome Research}

www.genome.org 
A

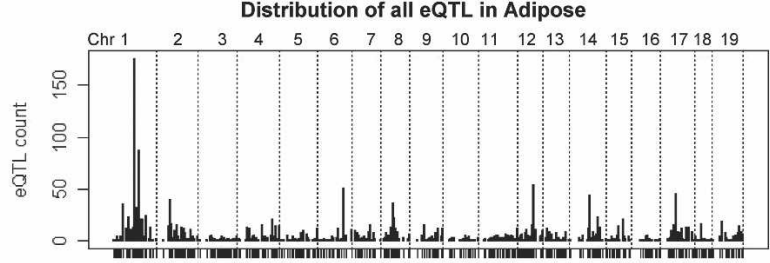

Distribution of eQTL for Sexually Dimorphic Genes in Adipose (>1.2 Fold)
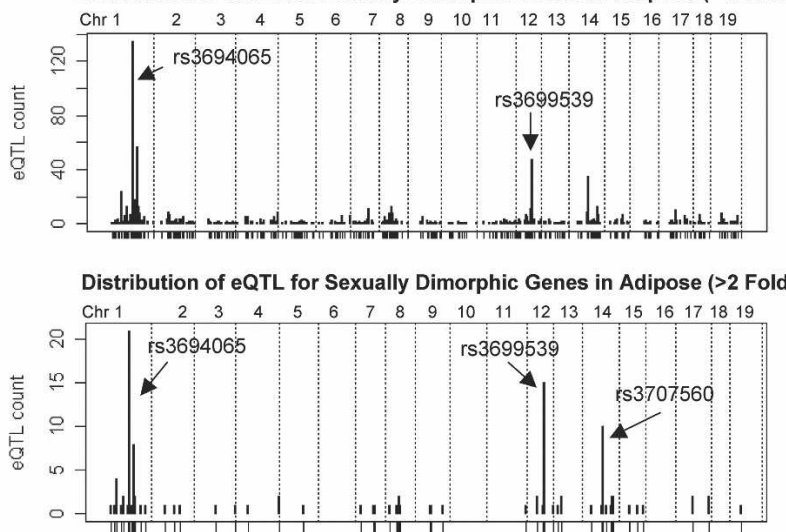

B
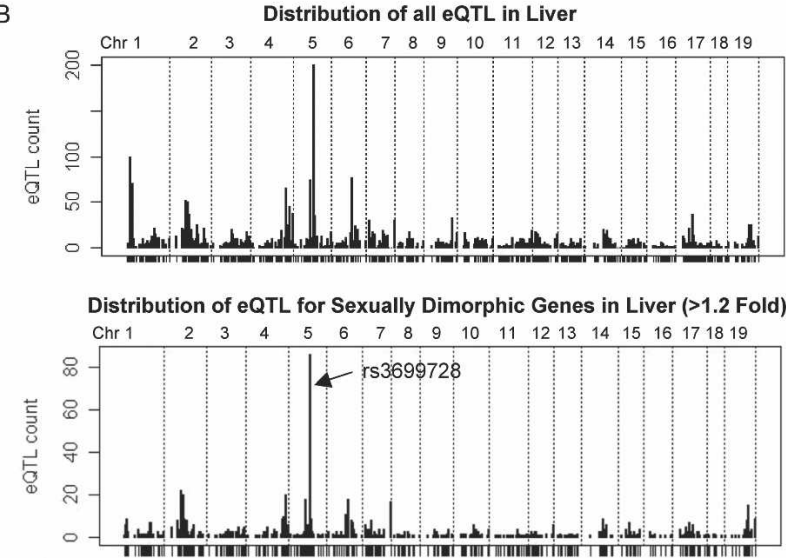

Figure 3. eQTL hotspots for sexually dimorphic genes in adipose and liver. (A) Genomewise distribution of significant eQTL for all active genes (upper) and sexually dimorphic genes at $>1.2$-fold-change level (middle) and $>2$-fold-change level (lower) in adipose tissue. (B) Genomewise distribution of significant $\mathrm{eQTL}$ for all active genes (upper) and sexually dimorphic genes at $>1.2$-fold-change level (lower) in liver. Hotspots with significantly enriched eQTL counts for the sexually dimorphic genes $(P<0.05$, Fisher's exact test with Bonferroni correction) are labeled.

these chromosomes (Arnold 2004). Interestingly, in addition to the $\mathrm{X}$ chromosome, which was more enriched for female-biased genes as suggested by (Khil et al. 2004), and the Y chromosome, which more commonly harbored male-biased genes, we also identified autosomes enriched with sexually dimorphic genes in a tissue-specific fashion. In several cases, the overrepresented autosomes appeared to contain sexually dimorphic genes with similar functionalities, thus supporting the presence of a connection between chromosome enrichment and function (Petkov et al. 2005).

The widespread sex differences in gene expression observed here are most likely due to differential effects of testicular and ovarian hormones (Becker et al. 2005; Amador-Noguez et al.
2005). Gonadal hormones contribute to sex differences both directly and indirectly and the indirect effects can be mediated by growth hormone (Legraverend et al. 1992; Arnold 2002; Wiwi and Waxman 2004). Despite the importance of hormones, an increasing amount of evidence supports a regulatory cascade concept of sexual dimorphism in gene expression; that is, the initiating events of sexual differentiation such as Sry expression trigger differential expression in many mediator genes that further regulate the sexually dimorphic expression of downstream genes (Krebs et al. 2003; Wiwi and Waxman 2004; Clodfelter et al. 2006). In addition to hormones and receptors, such mediators could include transcription factors and signaling molecules. Recently, the contributions of sex chromosome genes to sex differences in cell phenotype have been addressed (De Vries et al. 2002; Dewing et al. 2003; Arnold and Burgoyne 2004). In our study, several lines of indirect evidence also suggest a possible role of X- and Y-linked genes in the regulation of sexual dimorphism. In particular, there was a generalized sex-chromosome enrichment of the sexually dimorphic genes in all tissues examined, and a significant portion of common dimorphic genes across tissues are X- or Y-linked. Sex chromosome genes showing sex-biased expression in the present study may thus serve as candidate regulators.

Our TFBS and eQTL analyses provided evidence for tissuespecific regulation of the sexually dimorphic genes. Most overrepresented TFBS were distinct between tissues, suggesting that different transcription factors are involved in individual tissues. Six genetic loci were found to be regulatory hotspots for adipose dimorphic genes, and another 11 distinct loci were identified for dimorphic genes in liver, but no such regulatory loci were observed for muscle and brain.

To date, the regulation of sexually dimorphic genes has been studied in detail only in liver (Tullis et al. 2003; Waxman and Celenza 2003; Wiwi and Waxman 2004; Rinn and Snyder 2005; Clodfelter et al. 2006). Growth hormone-dependent transcription factors HNF4 (hepatocyte-enriched nuclear factor 4a; chromosome 2), HNF3 (chromosome 12), HNF6 (chromosome 9), and STAT5B (signal transducer and activator of transcription 5b; chromosome 11) and hormone-independent transcription factors RSL1 (regulator of sex-limitation 1; chromosome 13) and RSL2 (regulator of sex-limitation 2; chromosome 13) have been found to play important roles in the sex-biased expression of liver dimorphic genes. Among these, Foxa1 (formerly known as $H n f 3 A$ ) is located $\sim 6 \mathrm{cM}$ downstream from the liver hotspot at rs3686437 and Rsl1 is located within $5 \mathrm{cM}$ upstream of the hotspot at rs3681284. None of the known regulators are located close to the other nine eQTL hotspots for liver identified here. Interestingly, some individual loci controlled subsets of sexually dimorphic genes that were significantly enriched for particular functional categories. One male-biased gene with cis-eQTL at rs3699728, Btc (betacellulin, epidermal growth factor family members), may function as a candidate gene regulating the subset of sexually dimorphic genes involved in cell cycle and cell proliferation.

To our knowledge, this is the first report on genetic regulation of sexually dimorphic genes in adipose. Several promising candidate genes were located in the loci regulating sexual dimorphism in adipose. For example, Cebpe (CCAAT/enhancer binding protein C/EBP, $\varepsilon$ ) (Supplemental Table 9), a female-biased gene located upstream of marker rs 3707560 , is an important transcription factor required for normal function and development of macrophages and cytokine production (Tavor et al. 2002). Adi- 
pose sexually dimorphic genes with significant eQTL at this locus are involved in immune/defense/inflammatory response pathways. Five genes with cis-eQTL at this locus, Lgals3, Slc7a7, Dleu8 (previously known as 2610207P08Rik), Tep1, and Mett11d1 (previously known as D14Ertd209e), are also candidate genes. Smoc1 (SPARC related modular calcium binding 1), a female-biased gene with cis-eQTL at marker rs3699539, may control the subset of sexually dimorphic genes with eQTL at this locus.

In summary, we report comprehensive analyses of gene expression differences between sexes in four mouse somatic tissues. Thousands of sexually dimorphic genes identified were involved in tissue-specific biological functions and/or pathways relevant to common diseases and displayed tissue-specific chromosomal enrichment. Evidence for tissue-specific genetic and transcriptional regulation of the differentially expressed genes was also revealed, and several novel regulatory genetic hotspots were reported. These profiles should not only enhance our understanding of sex differences at the molecular level, but provide insights to our understanding of clinical traits such as neuropsychiatric disorders, atherosclerosis, obesity, and drug responses.

\section{Methods}

\section{$F_{2}$ progeny of B6.apoE $E^{-1-}$ and $\mathrm{C} 3 \mathrm{H} . \mathrm{apoE}^{-1-}$ intercross}

$\mathrm{C} 57 \mathrm{BL} / 6 \mathrm{~J}$ apoE null $\left(\mathrm{B} 6 . \mathrm{apoE}^{-/-}\right)$mice were purchased from the Jackson Laboratory, and $\mathrm{C} 3 \mathrm{H} / \mathrm{HeJ}$ apoE null $\left(\mathrm{C} 3 \mathrm{H} . \mathrm{apoE}^{-/-}\right)$mice were bred by backcrossing $\mathrm{B} 6 . \mathrm{apoE}^{-1-}$ to $\mathrm{C} 3 \mathrm{H} / \mathrm{HeJ}$ for 10 generations (Shi et al. 2000). All mice were fed ad libidum and maintained on a 12-h light/12-h dark cycle. $F_{1}$ mice were generated by constructing reciprocal crosses between B6.apoE ${ }^{-1-}$ and C $3 \mathrm{H}_{\text {.apoE }}{ }^{-1-}$, and $F_{2}$ mice were subsequently bred by intercrossing the $F_{1}$ mice. A total of $334 F_{2}$ mice (169 female, 165 male) were studied. They were fed Purina Chow (Ralston-Purina Co.) containing $4 \%$ fat until $8 \mathrm{wk}$ of age, then transferred to a Western diet (Teklad 88,137; Harlan Teklad) containing 42\% fat and $0.15 \%$ cholesterol for $16 \mathrm{wk}$, and sacrificed at $24 \mathrm{wk}$ of age. Mice were fasted for $4 \mathrm{~h}$ prior to euthanasia. Liver, gonadal adipose (epididymal fat pad in males, perimetrial fat pad in females), whole brain, and skeletal muscle (hamstring) were collected and immediately frozen in liquid nitrogen. The use of the apoE null background and high fat diet was to accelerate atherosclerosis, a sex-biased trait. We cannot rule out the influence of the particular genetic background and diet on the observations reported in this manuscript.

\section{RNA preparation and microarray analysis}

Tissues were homogenized and total RNA was extracted using Trizol reagent (Invitrogen) according to the manufacturer's recommendation. The control RNA pool was composed of equal aliquots of RNA derived from $150 F_{2}$ and parental samples. Portions of RNA were reverse-transcribed and labeled with either Cy3 or Cy5 fluorochrome. The microarrays contained 23,574 probes (60mer oligonucleotides) for mouse genes and ESTs, and 2186 control sequences (Agilent Technologies). Hybridization and transcript quantitation were performed as previously described (Schadt et al. 2003). Individual transcript intensities were corrected for experimental variation and normalized, and were reported as the mean $\log _{10}$ ratio (mlratio) of an individual experiment relative to a pool from the $F_{2}$ population (He et al. 2003). The microarray data from this study have been deposited to GEO under accession numbers GSE2814, GSE3086, GSE3087, and GSE3088.

\section{Selection of actively expressed genes}

The two-channel oligonucleotide microarray platform by Agilent used in our study is optimized to assess relative but not absolute expression levels. Therefore, in order to obtain a large yet stringent set of genes exhibiting biologically relevant expression activity in each of the tissues examined, we used a vigorous selection procedure. First we selected genes that showed significant differential expression (error-model derived $P<0.01$ ) (for details on the error-model, refer to He et al. 2003 and Weng et al. 2006) compared to a tissue-specific reference pool in at least $15 \%$ of the animals profiled. This is a common selection procedure used to restrict attention to the set of "most transcriptionally active genes" (Hughes et al. 2000; He et al. 2003; Schadt et al. 2003; Wang et al. 2006). To expand and enhance this transcriptionally active set of genes, we used an additional method that leveraged the multivariate nature of the data to assess differential expression activity. First, we identified genes with expression values that were highly correlated with the expression values of any of the "most transcriptionally active genes" $(P<10 \mathrm{e}-5$ for genegene Pearson correlation). We then identified genes in this correlated gene set that either (1) exhibited significant expression quantitative trait loci (at least one eQTL with a LOD score $>4.3$ or more than two eQTL with LOD scores $>3.0$ ) or (2) correlated with the characterized traits of adiposity, plasma lipids, or atherosclerosis ( $P<10 \mathrm{e}-5$ for gene-trait Pearson correlation) (S. Wang, E. Schadt, H. Wang, L. Ingram-Drake, W. Shi, T.A. Drake, and A.J. Lusis, in prep.). This additional selection procedure is based on measures that themselves indicate differential regulation, but that simultaneously consider all samples, thereby increasing the power over pointwise measures to identify genes exhibiting biologically relevant behavior. Expression QTL and correlation measures also fit with the intuitive notion that expressed and biologically relevant genes are likely under genetic regulation and correlate with one or more genes or phenotypic traits.

The genes that met all the above criteria were termed "active genes," representing actively expressed true signals based on our selection criteria. Of the 23,574 transcripts represented on the microarray, $12,845(54.5 \%)$ in liver, $16,664(70.7 \%)$ in adipose, 7367 (31.2\%) in muscle, and 4508 (19.1\%) in brain were identified as active genes and subsequently used as the starting gene sets for individual tissues in the subsequent analyses. It was important to select the active genes to correct for tissue-specific genomewise expression patterns when assessing tissue-specificity in sexually dimorphic gene expression. However, since we incorporated gene variance, gene-gene correlation, gene-trait correlation, and EQTL information in the selection criteria for reliability purposes, we undoubtedly excluded many genes, such as those that were less correlated with the phenotypic traits measured in the cross. This definitely contributed to the relatively small number of transcripts estimated to be expressed in brain.

\section{Identification of differentially expressed genes between male and female mice}

The mlratio of each active gene selected from the above procedure was used, and differential gene expression between male and female mice was analyzed using both the Student's $t$-test and the nonparametric Wilcoxon test. Genes that showed differential expression between sexes at $P<0.01$ from the Wilcoxon test were identified as sexually dimorphic genes. These genes were further divided based on several fold-change thresholds $(>1,>1.2,>2$, and $>3$ ) for additional analysis. FDR was assessed using the $\mathrm{Q}$ VALUE program (Storey and Tibshirani 2003) based on the $P$ values obtained from the Wilcoxon test for the experimental data. The minimum positive FDR for $P<0.01$ was reported.

\section{Genome Research}

www.genome.org 


\section{Analysis of enrichment for functional categories (Gene Ontology classifications)}

Each sexually dimorphic gene set was classified using Gene Ontology (GO) and Kyoto Encyclopedia of Genes and Genomes (KEGG) database assignments. EASE version 2.0 was used to identify overrepresented gene categories in each gene list. The significance level was determined by Fisher's exact test and Bonferroni correction for multiple category comparisons $(P<0.05)$. FDR was estimated using the built-in permutation option. The active genes in individual tissues were used as the population gene set for comparison.

\section{Analysis of chromosomal linkage}

Genes were localized based on the MGI database (http:// www.jax.org). The significance of any enrichment of a particular chromosomal location for the sexually dimorphic genes was evaluated using Fisher's exact test and Bonferroni correction for multiple chromosome comparisons embedded in EASE version $2.0(P<0.05)$. Additional Bonferroni correction for multiple tissues and multiple sex-bias groups was also conducted manually. The active genes in individual tissues were used as the population gene set.

\section{Analysis of enrichment for TFBS}

Each sexually dimorphic gene set was analyzed for enrichment of TFBS using the oPOSSUM program (Ho Sui et al. 2005; http:// www.cisreg.ca/cgi-bin/oPOSSUM/opossum). This program was chosen because of its advantage in processing thousands of genes simultaneously. For each transcript, the top $10 \%$ of conserved regions in the 5000-bp upstream/downstream sequences between mouse and humans with minimum conservation of $70 \%$ and matrix match threshold of $80 \%$ was scanned for TFBS in the JASPAR database using a position weight matrices algorithm. The highly enriched TFBS were identified using Fisher's exact test at $P<0.05$ by comparing the sexually dimorphic genes with the active genes in individual tissues. FDR was assessed using the $\mathrm{Q}$ VALUE program based on the $P$-values obtained from the oPOSSUM program. In the JASPAR database embedded in oPOSSUM, only the nonredundant and verified TFBS, which represented one-sixth of all known TFBS, were collected. Certain important TFBS such as estrogen receptor binding sites were missed.

\section{Identification of eQTL and regulatory hotspots}

The eQTL analysis was performed using a stepwise regression procedure that incorporated sex as a covariate in addition to the dominant and additive effects. We have recently described the detailed methodology and demonstrated the advantages of this approach (Wang et al. 2006). The sex-additive interaction term was considered when the additive effect was significant $(P<10 \mathrm{e}-$ 3 ), and the sex-dominant interaction term was included only if the sex-additive interaction term was significant. Significant and suggestive eQTL were identified at $P<5 \times 10$ e-5 and $P<1 \times 10$ e- 3 significance levels, respectively. The corresponding LOD thresholds were 4.3 (significant) and 3.0 (suggestive) using models with only significant additive and/or dominant terms, or 4.9 (significant) and 3.5 (suggestive) using models with significant additive effect and sex-additive interaction terms, or 5.4 (significant) and 4.0 (suggestive) using models with a significant additive effect as well as sex-additive and sex-dominant interaction terms. The number of significant and suggestive eQTL for both the sexually dimorphic genes and the active genes in the corresponding tissues was counted at each marker location. The enrichment of eQTL for sexually dimorphic genes compared to the active genes was calculated using Fisher's exact test. Hotspots were defined as the marker locations that had more than the average number of eQTL per marker location and that had significantly enriched eQTLs for the sexually dimorphic genes based on Fisher's exact test $(P<0.05$ after Bonferroni correction for multiple markers genomewise). Enrichment of functional categories and chromosome localization for genes with eQTL at the hotspot loci were analyzed as described above. Annotated genes located at $10 \mathrm{Mb}$ upstream and $10 \mathrm{Mb}$ downstream of the eQTL hotspots were obtained from NCBI build 35.1. Genes with ciseQTL were defined as those located within $20 \mathrm{Mb}$ upstream and downstream of the marker location on the same chromosome.

\section{Acknowledgments}

We thank Nadir Yehya, Debraj GuhaThakurta, Margarete Mehrabian, Eric Vilain, and Richard Davis for helpful discussions. This research was supported by research grants from the National Institutes of Health (HL30568, HL28481, and DK071673), the UCLA Laubisch fund, the Iris Cantor-UCLA Women's Health Center, and UCLA National Center for Excellence in Women's Health.

\section{References}

Amador-Noguez, D., Zimmerman, J., Venable, S., and Darlington, G. 2005. Gender-specific alterations in gene expression and loss of liver sexual dimorphism in the long-lived Ames dwarf mice. Biochem. Biophys. Res. Commun. 332: 1086-1100.

Anderson, G.D. 2002. Sex differences in drug metabolism: Cytochrome P-450 and uridine diphosphate glucuronosyltransferase. J. Gend. Specif. Med. 5: 25-33.

Arnold, A.P. 2002. Concepts of genetic and hormonal induction of vertebrate sexual differentiation in the twentieth century, with special reference to the brain. In Hormones, brain, and behavior (eds. W. Pfaff et al.), pp.105-135. Academic Press, San Diego, CA. . 2004. Sex chromosomes and brain gender. Nat. Rev. Neurosci. 5: 701-708.

Arnold, A.P. and Burgoyne, P.S. 2004. Are XX and XY brain cells intrinsically different? Trends Endocrinol. Metab. 15: 6-11.

Becker, J.B., Arnold, A.P., Berkley, K.J., Blaustein, J.D., Eckel, L.A., Hampson, E., Herman, J.P., Marts, S., Sadee, W., Steiner, M., et al. 2005. Strategies and methods for research on sex differences in brain and behavior. Endocrinology 146: 1650-1673.

Beyer, C., Kolbinger, W., Froehlich, U., Pilgrim, C., and Reisert, I. 1992. Sex differences of hypothalamic prolactin cells develop independently of the presence of sex steroids. Brain Res. 593: $253-256$.

Bieberich, C.J., Fujita, K., He, W.W., and Jay, G. 1996. Prostate-specific and androgen-dependent expression of a novel homeobox gene. $J$. Biol. Chem. 271: 31779-31782.

Clodfelter, K.H., Holloway, M.G., Hodor, P., Park, S.H., Ray, W.J., and Waxman, D.J. 2006. Sex-dependent liver gene expression is extensive and largely dependent upon signal transducer and activator of transcription 5b (STAT5b): STAT5b-dependent activation of male genes and repression of female genes revealed by microarray analysis. Mol. Endocrinol. 20: 1333-1351.

Depner, T.A. 2003. Prescribing hemodialysis: The role of gender. $A d v$ Ren. Replace. Ther. 10: 71-77.

DeVito, W.J. 1988. Distribution of immunoreactive prolactin in the male and female rat brain: Effects of hypophysectomy and intraventricular administration of colchicine. Neuroendocrinology 47: 284-289.

De Vries, G.J., Rissman, E.F., Simerly, R.B., Yang, L.Y., Scordalakes, E.M., Auger, C.J., Swain, A., Lovell-Badge, R., Burgoyne, P.S., and Arnold, A.P. 2002. A model system for study of sex chromosome effects on sexually dimorphic neural and behavioral traits. J. Neurosci. 22: 9005-9014.

Dewing, P., Shi, T., Horvath, S., and Vilain, E. 2003. Sexually dimorphic gene expression in mouse brain precedes gonadal differentiation. Brain Res. Mol. Brain Res. 118: 82-90.

Farsetti, A., Narducci, M., Moretti, F., Nanni, S., Mantovani, R., Sacchi, A., and Pontecorvi, A. 2001. Inhibition of ER $\alpha$-mediated trans-activation of human coagulation factor XII gene by 
heteromeric transcription factor NF-Y. Endocrinology 142: 3380-3388.

Hayden, J.M., Bergen, W.G., and Merkel, R.A. 1992. Skeletal muscle protein metabolism and serum growth hormone, insulin, and cortisol concentrations in growing steers implanted with estradiol-17 $\beta$, trenbolone acetate, or estradiol-17 $\beta$ plus trenbolone acetate. $J$. Anim. Sci. 70: 2109-2119.

He, Y.D., Dai, H., Schadt, E.E., Cavet, G., Edwards, S.W., Stepaniants, S.B., Duenwald, S., Kleinhanz, R., Jones, A.R., Shoemaker, D.D., et al. 2003. Microarray standard data set and figures of merit for comparing data processing methods and experiment designs. Bioinformatics 19: 956-965.

Ho Sui, S.J., Mortimer, J.R., Arenillas, D.J., Brumm, J., Walsh, C.J., Kennedy, B.P., and Wasserman, W.W. 2005. oPOSSUM: Identification of over-represented transcription factor binding sites in co-expressed genes. Nucleic Acids Res. 33: 3154-3164.

Hughes, I.A. 2001. Minireview: Sex differentiation. Endocrinology 142: 3281-3287.

Hughes, T.R., Marton, M.J., Jones, A.R., Roberts, C.J., Stoughton, R., Armour, C.D., Bennett, H.A., Coffey, E., Dai, H., He, Y.D., et al. 2000. Functional discovery via a compendium of expression profiles. Cell 102: 109-126.

Kahlert, S., Grohe, C., Karas, R.H., Lobbert, K., Neyses, L., and Vetter, H. 1997. Effects of estrogen on skeletal myoblast growth. Biochem. Biophys. Res. Commun. 232: 373-378.

Khil, P.P., Smirnova, N.A., Romanienko, P.J., and Camerini-Otero, R.D 2004. The mouse $X$ chromosome is enriched for sex-biased genes not subject to selection by meiotic sex chromosome inactivation. Nat. Genet. 36: 642-646.

Kobayashi, S., Isotani, A., Mise, N., Yamamoto, M., Fujihara, Y., Kaseda, K., Nakanishi, T., Ikawa, M., Hamada, H., Abe, K., et al. 2006. Comparison of gene expression in male and female mouse blastocysts revealed imprinting of the X-linked gene, Rhox5/Pem, at preimplantation stages. Curr. Biol. 16: 166-172.

Krebs, C.J., Larkins, L.K., Price, R., Tullis, K.M., Miller, R.D., and Robins, D.M. 2003. Regulator of sex-limitation (Rsl) encodes a pair of KRAB zinc-finger genes that control sexually dimorphic liver gene expression. Genes \& Dev. 17: 2664-2674.

Lauber, A.H., Romano, G.J., and Pfaff, D.W. 1991. Gene expression for estrogen and progesterone receptor mRNAs in rat brain and possible relations to sexually dimorphic functions. J. Steroid Biochem. Mol. Biol. 40: 53-62.

Lee, B.J., Cho, G.J., Norgren Jr., R.B., Junier, M.P., Hill, D.F., Tapia, V., Costa, M.E., and Ojeda, S.R. 2001. TTF-1, a homeodomain gene required for diencephalic morphogenesis, is postnatally expressed in the neuroendocrine brain in a developmentally regulated and cell-specific fashion. Mol. Cell. Neurosci. 17: 107-126.

Legraverend, C., Mode, A., Wells, T., Robinson, I., and Gustafsson, J.A 1992. Hepatic steroid hydroxylating enzymes are controlled by the sexually dimorphic pattern of growth hormone secretion in normal and dwarf rats. FASEB J. 6: 711-718.

Lusis, A.J., Taylor, B.A., Wangenstein, R.W., and LeBoeuf, R.C. 1983. Genetic control of lipid transport in mice. II. Genes controlling structure of high density lipoproteins. J. Biol. Chem. 258: 5071-5078.

Machleder, D., Ivandic, B., Welch, C., Castellani, L., Reue, K., and Lusis, A.J. 1997. Complex genetic control of HDL levels in mice in response to an atherogenic diet. Coordinate regulation of HDL levels and bile acid metabolism. J. Clin. Invest. 99: 1406-1419.

Mann, P.E. and Bridges, R.S. 2001. Lactogenic hormone regulation of maternal behavior. Prog. Brain Res. 133: 251-262.

McCormick, K.M., Burns, K.L., Piccone, C.M., Gosselin, L.E., and Brazeau, G.A. 2004. Effects of ovariectomy and estrogen on skeletal muscle function in growing rats. J. Muscle Res. Cell Motil. 25: 21-27.

McElreavey, K., Barbaux, S., Ion, A., and Fellous, M. 1995. The genetic basis of murine and human sex determination: A review. Heredity 75: $599-611$.

Paigen, B., Holmes, P.A., Mitchell, D., and Albee, D. 1987. Comparison of atherosclerotic lesions and HDL-lipid levels in male, female, and testosterone-treated female mice from strains $\mathrm{C} 57 \mathrm{BL} / 6, \mathrm{BALB} / \mathrm{c}$, and C3H. Atherosclerosis 64: 215-221.

Petkov, P.M., Graber, J.H., Churchill, G.A., Dipetrillo, K., King, B.L., and Paigen, K. 2005. Evidence of a large-scale functional organization of mammalian chromosomes. PLoS Genet. 1: e33.

Richards, S.M., Jensen, R.V., Liu, M., Sullivan, B.D., Lombardi, M.J.,
Rowley, P., Schirra, F., Treister, N.S., Suzuki, T., Steagall, R.J., et al. 2006. Influence of sex on gene expression in the mouse lacrimal gland. Exp. Eye Res. 82: 13-23.

Rinn, J.L. and Snyder, M. 2005. Sexual dimorphism in mammalian gene expression. Trends Genet. 21: 298-305.

Rinn, J.L., Rozowsky, J.S., Laurenzi, I.J., Petersen, P.H., Zou, K., Zhong, W., Gerstein, M., and Snyder, M. 2004. Major molecular differences between mammalian sexes are involved in drug metabolism and renal function. Dev. Cell 6: 791-800.

Saifi, G.M. and Chandra, H.S. 1999. An apparent excess of sex- and reproduction-related genes on the human X chromosome. Proc. Biol. Sci. 266: 203-209.

Schadt, E.E., Monks, S.A., Drake, T.A., Lusis, A.J., Che, N., Colinayo, V., Ruff, T.G., Milligan, S.B., Lamb, J.R., Cavet, G., et al. 2003. Genetics of gene expression surveyed in maize, mouse and man. Nature 422: 297-302

Shi, W., Wang, N.J., Shih, D.M., Sun, V.Z., Wang, X., and Lusis, A.J. 2000. Determinants of atherosclerosis susceptibility in the $\mathrm{C} 3 \mathrm{H}$ and C57BL/6 mouse model: Evidence for involvement of endothelial cells but not blood cells or cholesterol metabolism. Circ. Res. 86: $1078-1084$

Smith, M.J. 1994. Sex determination. Turning on sex. Curr. Biol. 4: $1003-1005$.

Spitzer, J.A. 1999. Gender differences in some host defense mechanisms. Lupus 8: $380-383$.

Storey, J.D. and Tibshirani, R. 2003. Statistical significance for genomewide studies. Proc. Natl. Acad. Sci. 100: 9440-9445.

Tavor, S., Vuong, P.T., Park, D.J., Gombart, A.F., Cohen, A.H., and Koeffler, H.P. 2002. Macrophage functional maturation and cytokine production are impaired in C/EBP $\varepsilon$-deficient mice. Blood 99: 1794-1801.

Taylor, B.A., Tarantino, L.M., and Phillips, S.J. 1999. Gender-influenced obesity QTLs identified in a cross involving the KK type II diabetes-prone mouse strain. Mamm. Genome 10: 963-968.

Tullis, K.M., Krebs, C.J., Leung, J.Y., and Robins, D.M. 2003. The regulator of sex-limitation gene, $r s l$, enforces male-specific liver gene expression by negative regulation. Endocrinology 144: 1854-1860.

Vawter, M.P., Evans, S., Choudary, P., Tomita, H., Meador-Woodruff, J., Molnar, M., Li, J., Lopez, J.F., Myers, R., Cox, D., et al. 2004 Gender-specific gene expression in post-mortem human brain: Localization to sex chromosomes. Neuropsychopharmacology 29: $373-384$.

Vilain, E. and McCabe, E.R. 1998. Mammalian sex determination: From gonads to brain. Mol. Genet. Metab. 65: 74-84.

Wang, W., Dong, L., Saville, B., and Safe, S. 1999. Transcriptional activation of E2F1 gene expression by 17ß-estradiol in MCF-7 cells is regulated by NF-Y-Sp1/estrogen receptor interactions. Mol. Endocrinol. 13: 1373-1387.

Wang, P.J., McCarrey, J.R., Yang, F., and Page, D.C. 2001. An abundance of X-linked genes expressed in spermatogonia. Nat. Genet. 27: 422-426.

Wang, S., Yehya, N., Schadt, E.E., Wang, H., Drake, T.A., and Lusis, A.J. 2006. Genetic and genomic analysis of a fat mass trait with complex inheritance reveals marked sex specificity. PLoS Genet. 2: e15.

Waxman, D.J. and Celenza, J.L. 2003. Sexual dimorphism of hepatic gene expression: Novel biological role of KRAB zinc finger repressors revealed. Genes \& Dev. 17: 2607-2613.

Weng, L., Dai, H., Zhan, Y., He, Y., Stepaniants, S.B., and Bassett, D.E. 2006. Rosetta error model for gene expression analysis. Bioinformatics 22: $1111-1121$

Wiwi, C.A. and Waxman, D.J. 2004. Role of hepatocyte nuclear factors in growth hormone-regulated, sexually dimorphic expression of liver cytochromes P450. Growth Factors 22: 79-88.

Wizemann, T.M. and Pardue, M. 2001. Exploring the biological contributions to human health: Does sex matter? National Academies Press, Washington, DC.

Xu, J., Watkins, R., and Arnold, A.P. 2005. Sexually dimorphic expression of the X-linked gene Eif2s3x mRNA but not protein in mouse brain. Gene Expr. Patterns 6: 146-155.

Received February 9, 2006; accepted in revised form May 22, 2006.

\section{Genome Research}

www.genome.org 


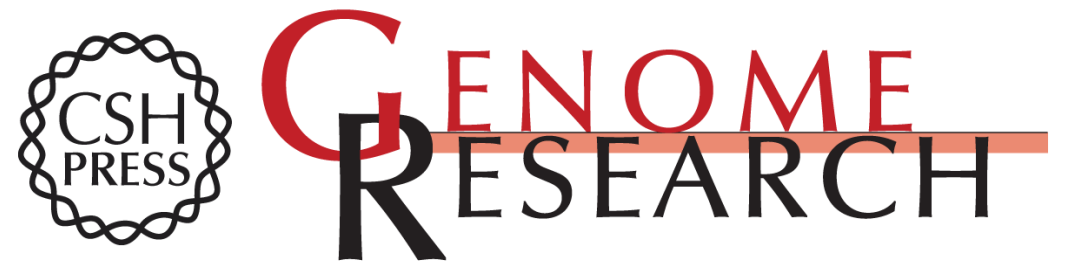

\section{Tissue-specific expression and regulation of sexually dimorphic genes in mice}

Xia Yang, Eric E. Schadt, Susanna Wang, et al.

Genome Res. 2006 16: 995-1004

Access the most recent version at doi:10.1101/gr.5217506

Supplemental http://genome.cshlp.org/content/suppl/2006/07/18/gr.5217506.DC1

Material

References This article cites 50 articles, 8 of which can be accessed free at: http://genome.cshlp.org/content/16/8/995.full.html\#ref-list-1

Open Access Freely available online through the Genome Research Open Access option.

License Freely available online through the Genome Research Open Access option.

Email Alerting Receive free email alerts when new articles cite this article - sign up in the box at the Service top right corner of the article or click here.

\section{Affordable, Accurate Sequencing.}

